Portland State University

PDXScholar

\title{
A Critical View of Helminthic Therapy: Is It a Viable Form of Treatment for Immune Disorders Under the Category of Inflammatory Bowel Disease?
}

Alyssa Murphy

Portland State University

Follow this and additional works at: https://pdxscholar.library.pdx.edu/honorstheses Let us know how access to this document benefits you.

\section{Recommended Citation}

Murphy, Alyssa, "A Critical View of Helminthic Therapy: Is It a Viable Form of Treatment for Immune Disorders Under the Category of Inflammatory Bowel Disease?" (2017). University Honors Theses. Paper 356.

https://doi.org/10.15760/honors.349

This Thesis is brought to you for free and open access. It has been accepted for inclusion in University Honors Theses by an authorized administrator of PDXScholar. Please contact us if we can make this document more accessible: pdxscholar@pdx.edu. 
Alyssa Murphy

Honors Thesis

Winter 2017

A Critical View of Helminthic Therapy: Is it a viable form of treatment for immune disorders under the category of inflammatory bowel disease?

\section{$\underline{\text { Abstract }}$}

In the developed world, Crohn's disease and colitis affects the lives of many individuals. Recently, a new form of treatment for these autoimmune diseases has gained recognition. This treatment uses helminths (Trichuris trichiura and Trichuris suis) as an immunomodulant to a human immune system. Various studies (Summers et. al., 2004; Dige et. al., 2016; Lopes et. al., 2016) have shown the safety and viability of this form of treatment, but many believe this area of research leaves much to be desired. Through this paper, the topic of helminthic therapy and its viability as a form of treatment for autoimmune diseases under the category of inflammatory bowel disease (IBD) will be discussed. A conclusion will be reached by reviewing these three key studies: Helminth Regulation of Immunity: A Three-pronged Approach to Treat Colitis by Lopes et. al., Trichuris suis therapy in Crohn's disease by Summers et. al., and Mucosal and systemic immune modulation by Trichuris trichiura in a self-infected individual by Dige et. al.

\section{$\underline{\text { Part 1: Helminths }}$}

Understanding the following research includes a knowledge of certain key terms. The terms "helminth" or "helminthic therapy" are casting an incredibly broad net. Helminth is a vague term meaning "worm". Pre-fixes are used to distinguish the different types of helminths; the main two being platy-helminth (flatworms) and nemat-helminth (roundworms) (Weinstock, 2012). Both species of helminths commonly used in helminthic therapy are nemat-helminth, and specifically whipworms. Trichuris trichiura (T.trichiura) is the human whipworm, and Trichuris suis (T.suis) is the pig whipworm (Cross, 1996). Focusing on the evolutionary paths and interactions with a human host for these two species of helminths will shed light on the viability of helminthic therapy for inflammatory bowel disease (IBD).

Parasites and parasitic roundworms have history that dates back to the beginning of man. 
Written record of parasitic infection is seen in Egyptian medicine, and recently a new section of science has developed called paleoparasitology (Cox, 2002). The discovery of helminth eggs in fossilized feces spurred the development of paleoparasitology, and has furthered understanding of the history of parasitic helminths. Through this look at the history of human-parasite interaction, various theories have arisen. Many believe we have "heirloom" parasites, which were inherited from our primate ancestors (Cox, 2002). Along with these passed down parasites, humans have picked up "souvenir" parasites while we spread across the globe. With the rise in global trading throughout human history, many parasites have been passed from one population of humans to another (Hawash et. al., 2016). This trading resulted in a wide spread of human parasites (about 300 species). Although humans are the hosts for these many parasites, with the rise of technology, many parts of the world rarely experience infection. The hygiene hypothesis is a theory that looks at the effect of this loss of parasitism on humans, and is part of the basis for researching helminthic therapy (Robinson and Bradley, 2010).

Its complex life cycle consists of six distinct stages (CDC, 2013). Beginning with unembryonated eggs being released through the feces (usually human), they then develop into a two cell organism and advance to a cleavage stage outside of their human host (CDC, 2013). Following these stages, the eggs become embryonated, and are able to start infecting their human host (CDC, 2013). The eggs are then ingested (through contact with infected soil), and once inside the host, the eggs will hatch in the small intestine and release larvae (CDC,2013). Once mature, the adult whipworm will attach itself in the ascending colon and cecum, and the females will start to produce eggs (CDC, 2013). Adults are around four centimeters in length, and can shed anywhere from 3,000 to 20,000 eggs per day, thus starting the cycle again (CDC, 2013). 


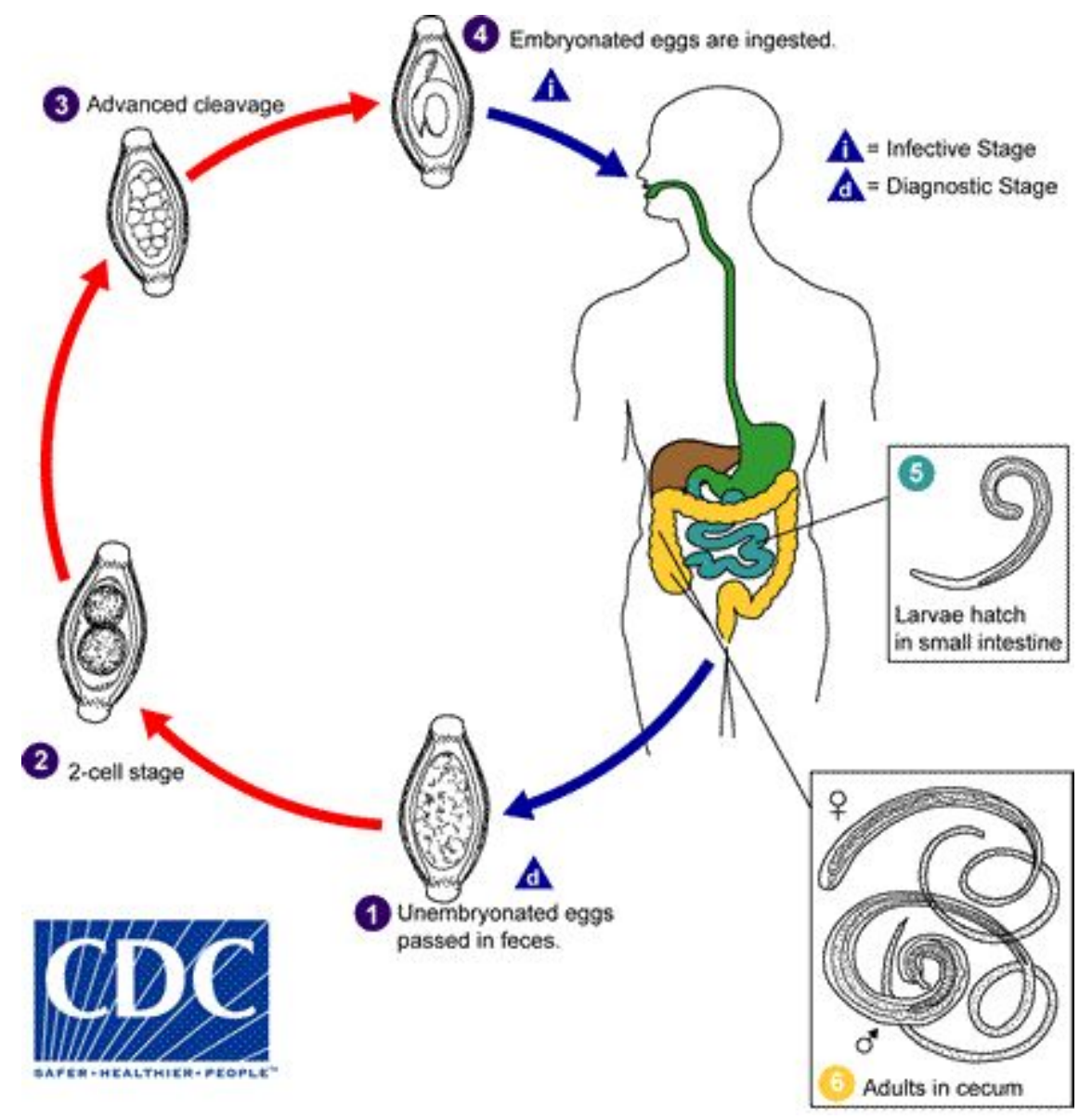

Figure 1: Life cycle of T. trichiura. (CDC, 2013)

In order to complete its life cycle, $T$. trichiura must advert the host's immune system. All helminths secrete large amounts of antigenic material, which many believe overloads the human immune system and renders it useless (Wakelin, 1996). Since T. trichiura (like many other helminths) is large and motile, it is physically able to withstand attacks from its host (from both the immune system and the high acidity of the stomach). Another explanation as to why $T$. trichiura is able to survive the human immune system is through the secretion of lymphocyte suppressor factors, which will reduce immune responsiveness (Wakelin, 1996). It is this explanation that is a major part of the basis for research of helminthic therapy, and why an infection of helminths could combat the symptoms of IBD. 
The helminth $T$. suis is also playing a part in helminthic therapy research. The life cycle of $T$. suis is almost identical to that of $T$. trichiura, except that it has co-evolved with the primary host as a pig (Pittman et. al., 2010). Because of this coevolution with pigs, T. suis is not as adept at avoiding a human immune attack as T. trichiura. However, like many other helminths, T. suis still secretes certain suppressants that lower immune responsiveness even in humans (Summers et. al., 2004). Since T. suis shares these immunomodulatory aspects with $T$. trichiura, but usually are unable to withstand a human environment for long, they make an optimal candidate for helminthic therapy research (Summers et. al., 2003).

\section{Part 2: The Immune System}

Another important part of this research is the role of the human immune system. Dissecting how the mucosal immune system in the digestive tract fends off non-self attackers, like T.suis or T.trichiura, and how is this process interacts with autoimmune disorders. The mucosal immune system is made of two distinct parts, the innate immune system and the adaptive immune system (NIH, 2003). For the purpose of this paper we will focus on the adaptive immune systems T-lymphocytes, also known as T-cells, due to the important part they play in the mucosal immune system (McGhee et. al., 1992).

The adaptive immune system is further divided into two sub-categories (humoral and cell-mediated). Within the humoral immune system, lymphocytes, specifically B-lymphocytes, are the main tracker cells. B-cells mature in the bone marrow, where they undergo rigorous trials to insure they are ready to produce the proper antibodies (making sure they will only produce antibodies that will tag non-self antigens) (Janeway et. al., 2001). Once matured they will travel to secondary lymphoid organs and await activation. Each B-cell is highly specific, meaning each 
will produce a specific antibody for a specific antigen (Janeway et. al., 2001). This works for the checks and balances part of the humoral immune system, allowing little error for over-reaction to other, similar, antigens. When a B-cell is alerted to a specific antigen, it begins to proliferate. Soon it will have numerous copies of itself, some being memory B-cells and others being effector B-cell (Janeway et. al., 2001). The memory B-cell will survive for decades to allow your immune system to have a faster and stronger defense towards this specific antigen, preventing symptoms from developing during secondary exposure to the pathogen (Janeway et. al., 2001). On the other side of this system are the effector B-cells. These B-cells will be the ones to poison the water supply, so to speak, becoming antibody factories and producing free-flowing antibodies that will mark antigens for phagocytosis, complement activation, neutralization or precipitation (Janeway et. al., 2001). These antibodies will allow macrophages, or other attacker cells to identify the pathogenic antigens.

T-cells fall into four categories, T-helper cells, T-regulatory cells, cytotoxic cells and memory cells. T-helper cells play an integral role in both cytoxic (CD-8) T cells and activating B lymphocytes for antibody production. T lymphocytes gain immunocompetence in the thymus through a process of clonal selection (Janeway et. al., 2001). In the immune system, T-helper cells act as an activator for the immune system (calling the troops to take up their arms). In a similar fashion to a B-cell, T-helper cells are incredibly specific and usually each T-helper cell is tuned to one specific antigen. This is again to help the immune system prevent cross-reactivity with other (possibly self) antigens. When a T-helper cell is presented its specific antigen by an antigen presenting cell (i.e. a B-cell, or dendritic cell, etc.), the T-helper cell will begin to sound the alarm (Janeway et. al., 2001). This includes proliferation of this specific T-helper cell, and 
some will become memory T-cells and others will become effector T-helper cells. Memory T-helper cells will stick around in the immune system (as was the case for memory B-cells), but the effector T-cells will rush into action. Effector T-cells will start to release cytokines, which signal other activated immune cells to ramp up production and get ready for battle (Janeway et. al., 2001). Cytokines are essentially proteins or polypeptides that act as a signaling switch for other activated immune cells (i.e. effector B-cells). Earlier in this discussion, it was mentioned that when B-cells become activated they proliferate. This activation is made possible and monitored by effector T-helper cells (Janeway et. al., 2001). This is another way the immune system is making sure it actually needs this large response to occur. With all of these ways that the immune system has to monitor itself, it seems there is little room for error or self-harm. However, in science there are usually exceptions to a rule, and for the immune system, autoimmune disorders are the exception.

Autoimmune diseases are still an elusive topic of research. Many health professionals and research scientists are not quite sure what causes certain autoimmune disorders, and have trouble coming up with cures or treatments for these diseases. When it comes to the umbrella term of inflammatory bowel disease (IBD), it is usually marked by a rise in the $T_{h} 1$ and $T_{h} 2$ immune response (Jackson et. al., 2008). $\mathrm{T}_{\mathrm{h}} 1$ and $\mathrm{T}_{\mathrm{h}} 2$ are sub-classifications of the $\mathrm{T}$-helper cells discussed earlier in this research, and both give rise to the signature symptom of IBD which is inflammation (Jackson et. al., 2008). When a patient is diagnosed with an autoimmune disease, their immune system works in a slightly different way than most. Where a healthy immune system would only mark and attack non-self antigens, an immune system with autoimmune tendency marks and attacks self-cells. In a simplified explanation, $\mathrm{T}_{\mathrm{h}} 1$ activate cytoxic cells and 
macrophages to attack self cells and $\mathrm{T}_{\mathrm{h}} 2$ cells activate $\mathrm{B}$ lymphocytes to produce antibodies against our own healthy cells (Jackson et. al., 2008). Normally, the body will release a $\mathrm{T}_{\mathrm{h}} 1$ immune response to an antigen for initial attack, and if it is able to kill and eliminate said antigen the body will return to equilibrium. If the antigen is not eliminated by the body's $\mathrm{T}_{\mathrm{h}} 1$ response, then the body will move to a $T_{h} 2$ response. $A T_{h} 2$ immune response will lead to widespread antibody production and a much more powerful response. This cycle between $T_{h} 1$ and $T_{h} 2$ immune responses is what causes the cyclic symptoms of $\operatorname{IBD}\left(\mathrm{Jackson}\right.$ et. al., 2008). The $\mathrm{T}_{\mathrm{h}} 1$ will start to attack self-cells, causing inflammation and other painful symptoms. If the "antigen" is not killed the body cycles into a $\mathrm{T}_{\mathrm{h}} 2$ immune response and starts to produce self-marking antibodies, which only proliferate the attack on self-cells (Jackson et. al., 2008). It was this knowledge of the $T_{h} 1$ and $T_{h} 2$ immune response that lead researchers to believe that certain helminths may be helpful in combating IBD.

The way helminths, like $T$. suis and $T$. trichiura, avoid the immune system is by secreting suppressor factors as stated earlier. These proteins depress the body's natural $\mathrm{T}_{\mathrm{h}} 1$ and $\mathrm{T}_{\mathrm{h}} 2$ immune responses, and therefore decrease the severity of these responses (Jackson et. al., 2008). Helminthic therapy research is based on the idea that a body with a heightened $T_{h} 1$ and $T_{h} 2$ immune responses could be regulated through the use of helminth infection, due to the fact that these lymphocyte suppressor factors down regulate the $T_{h} 1 / T_{h} 2$ immune response (Jackson et. al., 2008).

\section{Part 3A: Article Review}

In the article Helminth Regulation of Immunity: A Three-pronged Approach to Treat Colitis, Lopes et. al. brought forward three distinct forms of helminthic therapy to treat patients 
with ulcerative colitis. Lopes et. al. began their research with a few hypotheses that have driven helminthic therapy research. The "red queen hypothesis" states that as the helminth evolves better methods to evade its host's immune system, said host evolves more efficient ways of ejecting the parasite from its system (Lopes et. al., 2016). Another hypothesis Lopes et. al. reference was the "hygiene hypothesis", stating that a helminth could provide some type of protection against concomitant disease. These two hypotheses combined could explain why the helminth has been able to coevolve with the human species (Lopes et. al., 2016). As the two species (human and helminth) kept cycling through evolutionary tactics, the helminth presented protection against concomitant diseases. This could be seen as a health benefit to the host, and lead to a type of symbiotic lifestyle between host and parasite.

With these two hypotheses as a basis for research, Lopes et.al. presented these three forms of treatment for colitis: ingestion of viable ova or larvae of a helminth, use of crude helminthic extracts, and cellular immunotherapy. The most common type of helminthic therapy is through ingestion of viable ova or larvae, and through comparison of animal and human models it proves to be successful (Lopes et. al., 2016). Lopes et. al. discussed that in human models using T. suis ova or larvae there has been little to no side effects reported. Referencing studies that use both open label and placebo methods, Lopes et. al. provided adequate evidence of successful studies. However, Lopes et. al. state that there is a gap between animal (rodent) studies and human studies. This gap shows the need for further research of helminthic therapy using human patients.

Lopes et. al. also reviewed the use of helminth-derived extracts in treatment for colitis patients. Extracts could include things such as: soluble antigens from adult worms, egg antigens, 
or simply adult worm extracts (Lopes et.al., 2016). The use of these extracts negates the concerns of using live parasites for treatment of a patient with ulcerative colitis (Lopes et. al., 2016). Furthermore, these extracts could be used as blueprints for the development of new anti-inflammatory drugs (Lopes et. al., 2016). By reviewing a study featuring the use of Schistosoma mansoni (S. mansoni) egg extracts, Lopes et. al. showcased the success these extracts can have. However, in this same study, not all results were positive as certain types of colitis were not affected by the egg extracts (Lopes et. al., 2016). This lead the researchers. to propose that helminthic therapy research should be conducted with defined patient cohorts. Having a defined patient cohort, in this sense, would mean the patients with colitis being studied would all have similar types of colitis and similar symptoms in response to the colitis (Lopes et. al., 2016).

Thirdly, Lopes, et. al. proposed the use of cellular immunotherapy as a treatment for patients with colitis. Traditionally, cellular immunotherapy is used by transplanting cells into the patient in order to combat the disease. Lopes et. al. proposed transplanting cells from an individual infected with helminths to a patient diagnosed with colitis. The viability of this treatment has been shown through successful mice models, however there is a feasibility limitation when it comes to human patients (Lopes et. al., 2016). Even though there is this feasibility issue, it is shown that a helminth infection (or the antigens helminths produce) induce certain cell phenotypes that could be used for treatment of IBD (Lopes et. al., 2016).

Lopes et. al. concluded that research on treatments for IBD is urgently needed, due to the terrible toll colitis can take on patient's lives. Furthermore, through these three treatment methods Lopes et. al. determined a trend towards a personalized approach in patient care and 
treatment. Finally, Lopes et. al. concluded that there is definitely a link between host and parasite that may be beneficial to the treatment of IBD.

Helminth Regulation of Immunity: A Three-pronged Approach to Treat Colitis is a useful review to examine, because of its overview of three distinct treatment options. Many other studies focus their attention on the ingesting of live ova or larvae, and leave out the other options of cellular immunotherapy or helminthic extracts. Including these two other methods is inventive, and strengthens helminthic therapy research in various ways and broadens the field of research for helminthic therapy. Including results from various studies, and comparing those results between different treatment options gives a deeper insight into the gap in helminthic therapy research. Lopes, et. al.'s conclusions support helminthic therapy, especially in the sense of developing varying forms of treatment options. Some of the drawbacks of this article are based in the gap between human and animal trials. Throughout Helminth Regulation of Immunity: A Three-pronged Approach to Treat Colitis, Lopes, et. al. related the human trials to rodent trails that have had more conclusive results. Lopes, et. al. discussed that the less conclusive results in human trials are due to the lack in extensive research in this area. Although it seems likely that more research would close this gap in results, there may be other explanations as to why the human trials tend to be non-conclusive. One explanation could be the way trials are being conducted. Many of the studies using human trials do not have a defined patient cohort, which could be skewing their results. Lopes, et. al. mentioned the great necessity for defined patient cohorts in helminthic therapy research. Especially in the realm of cellular immunotherapy, the use of a more personalized care plan could drastically change the results of some of these studies. Because many autoimmune diseases vary from patient to patient, even 
when they have the same diagnoses, it makes more sense to vary the treatment method to fit each individual.

Defining a patient cohort for a study of helminthic therapy would involve finding patients with similar responses to a specific autoimmune disease. For example, when creating a human trial studying colitis patients, the researcher would need to decide on the type of response they would be looking into. If they decide to look at an immune response marked by heightened $\mathrm{T}_{\mathrm{h}} 2$ response, then they would need to test patient candidates for this response before including them in the study. This way, researchers can look at several different methods of helminthic therapy, and compare the results against one another to determine the best course of treatment. This extra setup step would allow researchers to tailor design a treatment method for patients with a specific immune response to colitis. Even though this would take more time, effort and money, it has the potential to change the lives of those living with IBD.

\section{Part 3B: Article Review}

Trichuris suis therapy in Crohn's disease is a 24-week open-label study on the safety and efficacy of ingesting T. suis ova as a treatment for Crohn's disease. Summers, et. al. (2004) conducted the study on patients with ages ranging from 18 to 72 years old, with a Crohn's disease activity index (CDAI) from 220 to 450. The CDAI is a form of measuring symptoms of Crohn's diseases, where 220 is moderately ill and 450 is severe disease (Summers et. al., 2004). A score is calculated by taking in various symptoms (i.e. number of liquid stool per day, abdominal pain, number of antidiarrheal drugs used) summed over the course of seven days, after adjustment by a weighting factor, and relating that score to an average (Summers et. al., 2004) (see table below). 


\begin{tabular}{|l|l|}
\hline \multicolumn{1}{|c|}{ Clinical or laboratory variable } & \multicolumn{1}{|c|}{$\begin{array}{c}\text { Weighting } \\
\text { factor }\end{array}$} \\
\hline Number of liquid or soft stools each day for seven days & $\times 2$ \\
\hline Abdominal pain (graded from 0-3 on severity) each day for seven days & $\times 5$ \\
\hline $\begin{array}{l}\text { General well-being, subjectively assessed from 0 (well) to 4 (terrible) each } \\
\text { day for seven days }\end{array}$ & $\times 7$ \\
\hline Presence of complications & $\times 20$ \\
\hline Taking Lomotil or opiates for diarrhea & $\times 30$ \\
\hline Presence of an abdominal mass $(0$ as none, 2 as questionable, 5 as definite) & $\times 10$ \\
\hline Hematocrit of $<0.47$ in men and $<0.42$ in women & $\times 6$ \\
\hline Percentage deviation from standard weight & $\times 1$ \\
\hline
\end{tabular}

Figure 2: CDAI data table. (Summers et. al., 2004)

Along with this age and CDAI range, the patients could be taking immunosuppressant drugs as long as it was kept constant. Patients were not allowed in the study if they filled any of the following criteria: they were taking immunomodulatory drugs; had previous treatments with antibiotic, antifungal, or antiparasitic medications; were diagnosed with diseases other than Crohn's disease; had undergone an ileostomy, colostomy, resection of greater than $50 \mathrm{~cm}$, or had obstructive symptoms; or were anticipating the need for surgery.

The method used to obtain T. suis ova included using pathogen free pigs, and ensuring the ova remained uncontaminated. First the pathogen free pigs were infected with $T$. suis ova, which were allowed to come to maturation. Once maturation was reached the eggs were collected from the colon, and cultured in vitro. The ova were embryonated in a phosphate buffered saline solution at $25^{\circ} \mathrm{C}$, and kept bacteria free through the use of $0.2 \% \mathrm{~K}_{2} \mathrm{Cr}_{2} \mathrm{O}_{7}$ solution 
and washed with sterile saline solution. Storing the ova at $5^{\circ} \mathrm{C}$ also ensured that there was no contamination by bacteria or other pathogens (Summers et. al., 2004). Through this process of obtaining T. suis ova, Summers et.al. showed that T. suis ova can stay viable, when stored properly, for up to nine months. This conclusion lends support to the potential for T. suis ova becoming a treatment which could be done by the patient at home with monthly checkups with a physician.

Through this 24-week open-label study, the following procedures were in use. The patients were given a dose of 2500 T.suis ova, suspended in liquid, every three weeks. At the time of entry and every six weeks, the following data points were collected: medical history, physical examination, pregnancy test, complete blood count, liver profile, stool examination checking for ova, pathogens, and Clostridium difficile toxin. Patients also kept diaries of their symptoms, and if a patient was taking immunosuppressant medication the dosage was kept constant. Summers et.al. also used the two-tail Fisher's exact test to examine characteristics that may indicate response or remission.

Results of this study support the viability and safety of using T. suis ova as a form of treatment for Crohn's disease. With $75.9 \%$ of patients experiencing response (decrease of $\mathrm{CDAI}>100$ or $\mathrm{CDAI}<150)$ and $65.5 \%$ of patients experiencing remission $(\mathrm{CDAI}<150)$ by week 12, this lends hard evidence to helminthic therapy being a viable form of treatment. Then by week $24,79.3 \%$ of patients had experienced response and $72.4 \%$ of patients experienced remission. These results partnered with the mean CDAI decrease being 195.1 by week 12 and 187.2 by week 24 , showing substantial evidence for the viability of helminthic therapy.

With these results, Summers, et. al. concluded that the use of $T$. suis ova was a safe and 
effective treatment for Crohn's disease. Since T. suis was not a natural parasite for humans, it lowered the risk of over-colonization of the patient (Summers et. al., 2004). This paired with the ability to control the source of the ova (pathogen-free pigs), ensured the patient's safety throughout the course of treatment. With an $80 \%$ response rate and a $73 \%$ remission rate, even in patients with refractory disease, the outcome of this study clearly shows significant evidence towards the viability of helminthic therapy (Summers et. al., 2004). However, with this study being an open-label study, it is impossible to rule out a placebo effect. This means that the results from this study justify further research (i.e. a blind clinical study), but cannot have truly conclusive results on its own.

The Summers et. al. study is important to look at, because of its unique use of the Crohn's disease activity index. Many other studies focus on a change from a cellular level, and do not look at patient's results holistically. By using the CDAI, Summers et. al. were able to show the change helminthic therapy was eliciting in their patient's lives. Also, this holistic approach resulted in a greater change over time than other studies focusing on change in certain interleukins or $T_{h} 1 / T_{h} 2$ response. While it is valuable to mark the clinical changes from the cellular level of a patient, it can be just as valuable to also look at the overall change in symptoms a patient is experiencing.

Trichuris suis therapy in Crohn's disease did support the viability and safety of helminthic therapy, and had substantial evidence behind this conclusion. Summers, et. al. produced viable results that were even better than predicted. Even with the possible placebo effect, Summers et. al. created a new way of viewing helminthic therapy research. With studies like these it is easy to see the need for further research, particularly blind studies, but it also 
showcases the real possibility for helminthic therapy to make a difference in the lives of those living with Crohn's disease.

\section{Part 3C: Article Review}

\section{Mucosal and systemic immune modulation by Trichuris trichiura in a self-infected}

individual is an open label study that consists of one self-infected patient (Dige et. al., 2016).

The purpose of this study was to determine if the helminth T. trichiura was able to

immunomodulate a human immune system. Through this study Dige et. al. could single out the

immunomodulant aspect of helminthic infect, and rule out the possible interaction of an intestinal disease.

Because this study consisted of a single self-infected patient with no prior intestinal disease, the methods used were slightly different than many other helminthic therapy studies. The patient was a 38 year-old with untreated psoriasis, but with no prior medical history of intestinal disease. Being $1.92 \mathrm{~m}$ tall and maintaining a constant body weight of $71 \mathrm{~kg}$, Dige et. al. determined the patient's daily fecal production to be $250 \mathrm{~g}$. This data point is necessary to determine the amount of $T$. trichiura ova needed to produce a moderate infection. After factoring in the amount of eggs that would actually hatch in the intestine (80\%) and the sex ratio (1:1), Dige et. al. decided that a dosage of 600 eggs would produce the infection needed. After nine weeks with no eggs in the patient's feces, the patient voluntarily took an extra three small doses of T. trichiura eggs. Throughout this infection period, the following data points were collected before and after infection: blood and faecal samples (biweekly), biopsies and ileo-colonoscopies at four locations (the terminal ileum, the cecum/ascending colon, the transverse colon and the sigmoid colon), and monitoring of the patient for signs of diarrhea, anemia, and weight loss. At 
the end of the study, the helminthic infection was terminated through the use of a mebendazole treatment.

The results Dige et. al. produced support the immunomodulatory effects of T. trichiura on a human immune system. This support was shown through a marked rise in T-cell cytokines that are directly linked to autoimmune diseases under the umbrella of IBD (i.e. $\left.T_{h} 1 / T_{h} 2\right)$. With a rise in T-cell cytokines, the patient's immune system lowered its $T_{h} 1$ response and raised its $T_{h} 2$ response. Dige et. al. conclude that this marked switch in T-cell response shows the ability of $T$. trichiura to be an immunomodulant for a human immune system. Another interesting result from this study was the patient developing Campylobacter colitis after the infection with T. trichiura. Dige et. al. concluded that an infection with helminths may leave the human immune system open to infection with other bacteria. Through this finding, Dige et. al. suggest that future studies ensure the patients have no prior infection of Campylobacter, and that the ova being ingested are free of contaminants.

Mucosal and systemic immune modulation by Trichuris trichiura in a self-infected individual is an article that brings an entirely new perspective to the study of helminthic therapy. Through the use of a patient with no prior intestinal disease, Dige et. al. were able to produce results that negate the coincidental changes in disease activity. Focusing on only the interaction between $T$. trichiura and the human immune system allowed Dige et. al. to produce results that support the immunomodulatory effects helminths can have on humans. Having conclusive evidence of this interaction could further helminthic therapy research, and supports the hygiene hypothesis. Through this study Dige et. al. also found that helminths may leave the human immune system vulnerable to co-infection. Even though this result seems negative for the use of 
helminthic therapy, it was able to provide instruction for future studies. Through this study conducted by Dige et. al., helminthic therapy research was furthered by the knowledge of the immunomodulatory effects of $T$. trichiura has on a human immune system, and given valuable instruction to avoid co-infection of Campylobacter.

\section{Part 4: Conclusion}

In conclusion, treatment of autoimmune diseases categorized as inflammatory bowel disease (IBD) with infection of helminths (T. suis) is both safe and effective. Though more research is needed, evidence of the viability of helminthic therapy is clearly shown in the studies discussed in this paper. Researchers Lopes et. al. in Helminth Regulation of Immunity: A Three-pronged Approach to Treat Colitis support the use of helminths as a treatment for IBD in various forms. These forms include helminth extracts, cellular immunotherapy, and the most common method of ingesting live ova or larvae (Lopes et. al., 2016). Presenting evidence that each of these three forms have produced successful results (patients gaining remission status, or positively responding to treatment) in clinical trials, expands helminthic therapy research to include these innovative methods.

In Summers et. al.'s research, Trichuris suis therapy in Crohn's disease, supporting evidence was shown through tracking patient progress using the Crohn's disease activity index (CDAI). Using the CDAI to determine the viability of helminthic therapy was a unique way of examining results, and provided a holistic approach to Summers et. al.'s research. Their results were positive, with a majority of their patients gaining remission or at least responding positively to the treatment (CDAI decreasing by at least 100 points) (Summers et. al., 2004). Through this supporting evidence, Summers et. al. were able to provide a new way of examining results of 
helminthic therapy studies.

Dige et. al., authors of Mucosal and systemic immune modulation by Trichuris trichiura in a self-infected individual, also presented concrete results supporting the immunomodulatory effects helminths have on a human immune system. By studying a self-infected individual with no prior intestinal disease, Dige et. al. were able to rule out the possibility of coincidental changes in disease activity in their results. Having results like these presents concrete evidence of the immunomodulatory influence helminths can have on a patient's immune system (Dige et. al., 2016). This study solidifies the basis of helminthic therapy research, and warns future research of things to avoid.

After reviewing these three studies, helminthic therapy seems to be a safe and effective form of treatment. However, in science, nothing is ever truly proven and research must always be followed by more research. For future helminthic therapy research, the following points should be taken into consideration. When creating a clinical study, patients should be chosen from a defined cohort. A defined patient cohort would limit the influencing variables in a study, therefore the results would be more conclusive (Lopes et. al., 2016). Future studies should also use the CDAI to examine results, and pair this with determining results from a cellular level. This would allow studies to view their data comprehensively, thus solidifying their results. Finally, future research should ensure a lack of contamination when using live ova or larvae. This is important, because helminths can leave the human immune system vulnerable to bacterial infection. 


\section{Bibliography}

Cross JH. (1996). Enteric Nematodes of Humans. In: Baron S, editor. Medical Microbiology. 4th edition. Galveston (TX): University of Texas Medical Branch at Galveston. Chapter 90. Available from: https://www.ncbi.nlm.nih.gov/books/NBK8261/

Czaja, A. J. (2014). Review article: the prevention and reversal of hepatic fibrosis in autoimmune hepatitis. Alimentary Pharmacology \& Therapeutics, 39(4), 385-406. doi:10.1111/apt. 12592

Dige A, Rasmussen TK, Nejsum P, et al. Mucosal and systemic immune modulation by Trichuris trichiurain a self-infected individual. Parasite Immunology. October 2016.

doi:10.1111/pim.12394.

Hawash, M. B., Betson, M., Al-Jubury, A., Ketzis, J., LeeWillingham, A., Bertelsen, M. F., . . . Nejsum, P. (2016, January 22). Whipworms in humans and pigs: origins and demography. Retrieved March 11, 2017, from https://parasitesandvectors.biomedcentral.com/articles/10.1186/s13071-016-1325-8\#Sec13

Janeway CA Jr, Travers P, Walport M, et al. Immunobiology: The Immune System in Health and Disease. 5th edition. New York: Garland Science; 2001. The mucosal immune system. Available from: https://www.ncbi.nlm.nih.gov/books/NBK27169/

K. Robinson and J. E. Bradley, The allergy epidemic: can helminths supply the antidote?, 2010 (40) 1586-1589.

Kronenberg, M., \& Havran, W. L. (2014). Immunology: Oiling the wheels of autoimmunity. Nature, 506(7486), 42-43. doi:10.1038/506042a

Lopes, Fernando PhD, Matisz, Chelsea MSc, Reyes, José L. PhD, et al. Helminth Regulation of Immunity: A Three-pronged Approach to Treat Colitis. Inflammatory Bowel Diseases. October 2016. doi:10.1097/MIB.0000000000000889.

Maizels RM, McSorley HJ. Regulation of the host immune system by helminth parasites. The Journal of Allergy and Clinical Immunology. 2016;138(3):666-675.

doi:10.1016/j.jaci.2016.07.007.

Moglia Willis, S. (2016). The Role of Diet in the Prevention and Treatment of Autoimmune Diseases. Access, 30(3), 16-26. 
Nutrition Is Key Against Autoimmunity. (2011). Environmental Nutrition, 34(11), 1-6.

Parker GA, Chubb JC, Ball MA, Roberts GN. Evolution of complex life cycles in helminth parasites. Nature. 2003;425(6957):480-484. doi:10.1038.

Pritchard D, Blount D, Schmid-Grendelmeier P, Till S. Parasitic worm therapy for allergy: Is this incongruous or avant-garde medicine?. Clinical \& Experimental Allergy [serial online]. April 2012;42(4):505-512. Available from: Health Source: Nursing/Academic Edition, Ipswich, MA. Accessed October 21, 2016.

Puentes, F., Dickhaut, K., Hofstätter, M., Pfeil, J., Lauer, U., Hamann, A., ... \& Rötzschke, O. (2016). Immune modulation and prevention of autoimmune disease by repeated sequences from parasites linked to self antigens.Journal of Neuroimmune Pharmacology, 1-14.

Rose, N. R. (2016). Prediction and Prevention of Autoimmune Disease in the 21st Century: A Review and Preview. American journal of epidemiology, kwv292.

Treatment Trials For Crohn's Disease and Ulcerative Colitis. (2002). Ostomy Quarterly, 39(3), 28.

The Facts About Inflammatory Bowel Diseases - ccfa.org. Crohn's and Colitis Foundation of America. http://www.ccfa.org/assets/pdfs/updatedibdfactbook.pdf. Accessed November 13, 2016.

Trichuriasis. (2013, November 29). Retrieved January 22, 2017, from https://www.cdc.gov/dpdx/trichuriasis/index.html

Weinstock, J. V. (2012). The worm returns: Joel V. Weinstock explains why several clinical trials are deliberately infecting people with helminths to treat autoimmune diseases. Nature, 491(7423), 183-185. http://doi.org/10.1038/491183a 